\title{
GROWTH AND FAT MASS IN PRETERM INFANTS FED A PROTEIN-ENRICHED POSTDISCHARGE FORMULA (PDF): A RANDOMIZED CONTROLLED TRIAL
}

\author{
P. Roggero, M.L. Giannì, O. Amato, N. Liotto, L. Morlacchi, A. Orsi, P. Piemontese, F. Taroni, F. Mosca \\ Fondazione IRCCS Ca' Granda Ospedale Maggiore Policlinico, NICU, University of Milan, Milan, Italy
}

Background and aims: Male infants with $\mathrm{BW}<1250 \mathrm{~g}$ benefit from PDF. Fetal growth seems to influence growth recovery whereas fat restoration occurs irrespective of BW. To evaluate whether being fed a PDF determines a growth benefit in two subgroups of infants.

Methods: 123 preterm infants born AGA (BW=1193.4 $\pm 230 \mathrm{~g}$; $\mathrm{GA}=29 \pm 1.9$ wks) and 84 born SGA $(\mathrm{BW}=1127 \pm 262 \mathrm{~g} ; \mathrm{GA}=31.3 \pm 1.9 \mathrm{wks})$ were randomized at term corrected age (CA) in G1: 59 AGA fed PDF $(2.9 \mathrm{~g} / 100 \mathrm{kcal}), \mathrm{G} 2: 64$ AGA fed term formula (TF) $(2.1 \mathrm{~g} / 100 \mathrm{kcal}), \mathrm{G} 3: 41$ SGA fed PDF, G4: 43 SGA fed TF. From 6 months infants were fed a follow on formula and weaned according to ESPGHAN recommendations. Growth and body composition were assessed by an air displacement plethysmography system at term, 1, 3, 5, 6, 12 months. ANOVA, regression analysis.

Results: G1 and G3 protein intakes were higher than those of $G 2(p<0.005)$ and $G 4(p<0.05)$, respectively, whereas weight, length and fat mass were similar at each study point. $\mathrm{G} 1$ mean $\mathrm{HC}(\mathrm{cm})$ was bigger than that of $\mathrm{G} 2$ at six months $(43.5 \pm 1.9$ vs $42.6 \pm 1.6, \mathrm{p}=0.03)$ whereas at 12 months no difference was found (45.4 \pm 1.6 vs $46 \pm 1.6$ ). In AGA infants being fed a PDF formula, being male, not having a postnatal growth retardation at term correlated with bigger $\mathrm{HC}$ at six months $[(\mathrm{p}<0.001)$, unstandardized $\mathrm{B}$ coefficient (SE) $0.9(0.36) ; 1.2(0.36) ; 1.2(0.37)$, respectively].

Conclusions: Male AGA without postnatal growth retardation at term but not SGA infants appear to benefit from being fed PDF. 